\title{
Characteristics and effectiveness of preoperative consultations in a tertiary hospital
}

\author{
Hyesun Paik, Chae-Won Lim, and Ho-Geol Ryu \\ Department of Anesthesiology and Pain Medicine, Seoul National University Hospital, Seoul, Korea
}

As the population is aging and chronically-ill patients have increased, the need of consultation has also increased [1]. And, as medical specialties become even more specialized, the dependency on consultation in patient evaluation and management has elevated. Appropriate use of consultation services might result in better prognosis for postoperative patients. However, overutilization of consultation services can cause unnecessary increase in hospitalization periods, efforts, and resources. The purpose of this study is to evaluate the current status of preoperative consultations and its effectiveness.

The medical records of patients who had taken the elective operations for general anesthesia, regional anesthesia and nerve block in March and April of 2010 at the tertiary hospital's operating theater were being reviewed. The number of consultations, consulted departments, the consulting surgical departments, and number of operations conducted by each surgical department were being analyzed. The total number of consultations in the hospital during the study period was retrieved, and consultation proportion was calculated by number of consultations per number of operations.

The number of requested consultations was classified according to the specialties consulted. The purpose of consultation was specified as 'for risk evaluations,' 'for perioperative management', or for both. The consultation responses was classified into 'suggesting to proceed the operation with attention', 'suggesting to proceed with the operation after additional work-ups' and 'suggesting to delay the operation'.

As a result, in March and April, 2010, the total number of anesthesia, which includes general or regional anesthesia and nerve block, was 1245 . Of all the operations, 453 patients (36.4\%) had been requested for consultation, and the total number of consultations taken was 663 .

More preoperative consultations had been conducted by orthopedic surgery, followed by otolaryngology, urosurgery, general surgery. For cases of specialty consultation, cardiology (271 cases, $40.9 \%$ ) is the leading specialty followed by pulmonology (129 cases, 19.5\%), endocrinology (86 cases, 13.0\%), neurology (66 cases, 9.9\%). The number of consultations for anesthesiology is only $14(2.1 \%)$ (Table 1$)$.

The aim of consultation was mainly for perioperative managements (54.6\%) and preoperative risk evaluations (37.0\%). As a result of consultation, additional evaluation was recommended for $13.6 \%$, and delaying operation was recommended for $1.1 \%$ (Table 1).

This result shows that about one third of patients who were scheduled for surgery had been requested for preoperative con-

Table 1. The Specialty Requested Consultations and Results for Consultations

\begin{tabular}{lccc}
\hline Specialty & $\begin{array}{c}\text { Number of } \\
\text { consultations }\end{array}$ & $\begin{array}{c}\text { Recommended } \\
\text { additional } \\
\text { work-ups }\end{array}$ & $\begin{array}{c}\text { Recommended } \\
\text { delaying } \\
\text { operations }\end{array}$ \\
\hline Cardiology & 271 & 36 & 6 \\
Pulmonology & 129 & 20 & 0 \\
Endocrinology & 86 & 5 & 0 \\
Neurology & 66 & 11 & 0 \\
Anesthesiology & 14 & 0 & 0 \\
Etc. & 97 & 18 & 1 \\
Total & 663 & 90 & 7 \\
\hline
\end{tabular}

Corresponding author: Ho-Geol Ryu, M.D., Department of Anesthesiology and Pain Medicine, Seoul National University Hospital, 101, Daehakro, Jongno-gu, Seoul 110-744, Korea. Tel: 82-2-2072-2065, Fax: 82-2-747-5639, E-mail: hogeol@gmail.com

(c) This is an open-access article distributed under the terms of the Creative Commons Attribution Non-Commercial License (http:// creativecommons.org/licenses/by-nc/3.0/), which permits unrestricted non-commercial use, distribution, and reproduction in any medium, provided the original work is properly cited. 
sultations, and the total number was 663 . However, the majority of consultations did not have an impact. Of the 663 consultations, 90 consultations suggested for further evaluations and 7 consultations suggested to delaying the scheduled operations.

Overutilization of consultation results in a waste of time and resources. It might be originated from vague understanding of consultation procedures. In a previous retrospective review of 202 cardiology consultations at a university hospital, it was found that only 108 asked for evaluations, 79 asked for a clearance, and 9 did not specifically ask for anything [2]. Our study showed similar results. A large percentage of consultations had no effects on pre-operative evaluations or managements for patients.

Nearly half of consultations were requested to cardiologists, and only $5.4 \%$ of them were asked for further work-ups. Majority of requested consultations were responded based on the American College of Cardiology-American Heart Association (ACC-AHA) guidelines. This suggests that cardiology consulta- tions may be overutilized and even abused to some extent.

In this circumstance, the role of anesthesiologist on pre-operative evaluations should be emphasized. Our study showed that anesthesiology was consulted in only $2.1 \%$, although anesthesiology specializes in perioperative care. According to a previous study, assigning the anesthesiologist with the responsibilities of deciding which patients need further evaluation has been associated with a $73 \%$ decrease in preoperative consultations [3]. Another study reports that the anesthesiology decision of "patient unfit for operation and anesthesia" has a high accuracy, and that preoperative medical managements significantly reduces such complications [4].

In conclusion, current consultation system tends to be excessively used, and the roles of anesthesiologist for preoperative evaluations and managements need to be re-evaluated. Physicians should not try to consult with vague intentions, and anesthesiologists should also not suggest unnecessary consultations to physicians.

\section{References}

1. Wijeysundera DN, Austin PC, Beattie WS, Hux JE, Laupacis A. A population-based study of anesthesia consultation before major noncardiac surgery. Arch Intern Med 2009; 169: 595-602.

2. Kleinman B, Czinn E, Shah K, Sobotka PA, Rao TK. The value to the anesthesia-surgical care team of the preoperative cardiac consultation. J Cardiothorac Anesth 1989; 3: 682-7.

3. Fischer SP. Development and effectiveness of an anesthesia preoperative evaluation clinic in a teaching hospital. Anesthesiology 1996; 85: 196-206.

4. Prause G, Ratzenhofer-Komenda B, Smolle-Juettner F, Krenn H, Pojer H, Toller W, et al. Operations on patients deemed "unfit for operation and anaesthesia": what are the consequences? Acta Anaesthesiol Scand 1998; 42: 316-22. 